\title{
DECOMPOSITIONS OF MODULES INTO PROJECTIVE MODULES AND CS-MODULES
}

\author{
Somyot PlubTieng
}

\begin{abstract}
Let $M$ be a right $R$-module. It is shown that $M$ is a locally Noetherian module if every finitely generated module in $\sigma[M]$ is a direct sum of a projective module and a CS-module. Moreover, if every module in $\sigma[M]$ is a direct sum of a projective module and a CS-module, then every module in $\sigma[M]$ is a direct sum of modules which are either indecomposable projective or uniform $\sum$-quasi-injective. In particular, if every module in $\sigma[M]$ is a direct sum of a projective module and a quasi-continuous module, then every module in $\sigma[M]$ is a direct sum of a projective module and a quasi-injective module.
\end{abstract}

\section{INTRODUCTION}

A module $M$ is called a CS-module (or extending module [5]) if every submodule of $M$ is essential in a direct summand of $M$. CS-modules provide a useful generalisation of (quasi-)injective modules and (quasi-)continuous modules (see [11]). The study of rings over which finitely generated right modules are CS was initiated by Dung and Smith [4]. It was shown further in Huynh, Rizvi and Yousif [9] and Vanaja [12] that such rings must be right Noetherian. Huynh and Rizvi [10] recently investigated rings over which every countably generated right module is a direct sum of a projective module and a CS-module, and they showed that these rings form a special class of right Artinian rings. They gave also several characterisations of rings over which every (countably generated) right $R$-module is a direct sum of a projective module and a quasi-continuous module.

In this paper, we use module-theoretic methods to consider the related properties in more general settings. First, we show that a module $M$ is locally Noetherian if every finitely generated module in $\sigma[M]$ is a direct sum of a projective module and a CS-module. Further, we study the modules $M$ satisfying the stronger property that every module in $\sigma[M]$ is a direct sum of a projective module and a CS-module. We show that such modules $M$ turn out to be pure semisimple in the sense of Wisbauer [13, Section 53], and

Received October, 1999

I would like to thank Professor Nguyen Viet Dung for pointing out Proposition 3 and for his several helpful suggestions. Moreover I wish to express my thanks to Professor Sompong Dhompongsa for drawing my attention to the subject, and for many useful discussions.

Copyright Clearance Centre, Inc. Serial-fee code: 0004-9727/00 \$A2.00+0.00. 
every module in $\sigma[M]$ is a direct sum of indecomposable projective modules and uniform $\sum$-quasi-injective modules. As a consequence, we deduce that if every module in $\sigma[M]$ is a direct sum of a projective module and a quasi-continuous module, then every module in $\sigma[M]$ is a direct sum of a projective module and a quasi-injective module. Specialising to the special case when $M_{R}=R_{R}$, our results provide new additional information on certain classes of Artinian rings studied recently by Huynh and Rizvi [10].

\section{THE RESULTS}

Throughout this paper we consider associative rings $R$ with identity and unitary right $R$-modules. For a right $R$-module $M, \sigma[M]$ will denote the category of all right $R$-modules which are submodules of $M$-generated modules. For basic definitions and properties of rings, modules and categories we refer to Anderson and Fuller [1] and Wisbauer [13].

We shall consider the following two conditions on a right $R$-module $M$ :

(*) Every finitely generated module in $\sigma[M]$ is a direct sum of a projective module and a CS-module;

(**) Every module in $\sigma[M]$ is a direct sum of a projective module and a CSmodule.

We start our investigation by proving the following result.

THEOREM 1 . Let $M$ be a right $R$-module satisfying (*). Then $M$ is locally Noetherian.

Proof: Let $M$ be a right $R$-module satisfying (*) and let $N$ be a finitely generated submodule of $M$. We first aim to show that $N / \operatorname{Soc}(N)$ is Noetherian. Let $E$ be an essential submodule of $N$, and set $K=N / E$. Then $K$ is a singular module. Clearly every finitely generated module in $\sigma[K]$ can not contain nonzero projective submodules. Thus, by $(*)$, every finitely generated module in $\sigma[K]$ is CS. Then, by [9, Theorem 5], it follows that $K$ is Noetherian. Therefore, $N$ has ACC on essential submodules, hence $N / \operatorname{Soc}(N)$ is Noetherian by [5, Theorem $5.15(1)]$.

We show now that $\operatorname{Soc}(N)$ is finitely generated, which would imply that $N$ is Noetherian. Assume on the contrary that $\operatorname{Soc}(N)$ is infinitely generated. Then we may write Soc $(N)=H_{1} \oplus H_{2}$, where $H_{1}$ and $H_{2}$ are infinite direct sums of simple modules.

By hypothesis, we have $N / H_{1}=\overline{P_{1}} \oplus \overline{Q_{1}}$ where $\overline{P_{1}}$ is a projective module and $\overline{Q_{1}}$ is a CS-module. Let $Q_{1}$ be the inverse image of $\overline{Q_{1}}$ in $N$. Then clearly $\overline{P_{1}} \simeq N / Q_{1}$, and $Q_{1} / H_{1}$ (being isomorphic to $\overline{Q_{1}}$ ) is a CS-module. Since $\overline{P_{1}}$ is projective, $N=Q_{1} \oplus Q_{2}$ for some submodule $Q_{2}$ of $N$. Then $\operatorname{Soc}(N)=\operatorname{Soc}\left(Q_{1}\right) \oplus \operatorname{Soc}\left(Q_{2}\right)$.

Observe that, because $\overline{Q_{1}} / \operatorname{Soc}\left(\overline{Q_{1}}\right)$ is Noetherian by the above argument, and $\overline{Q_{1}}$ is a finitely generated CS-module, it follows from [5, Lemma 9.1] that $\operatorname{Soc}\left(\overline{Q_{1}}\right)$ is finitely 
generated. Hence $\overline{Q_{1}}$, and so $Q_{1} / H_{1}$, has finite uniform dimension. Therefore, this clearly implies that $\operatorname{Soc}\left(Q_{2}\right)$ is infinitely generated.

Note that

$$
N / \operatorname{Soc}(N) \simeq\left(Q_{1} / \operatorname{Soc}\left(Q_{1}\right)\right) \oplus\left(Q_{2} / \operatorname{Soc}\left(Q_{2}\right)\right),
$$

where $Q_{1} \neq \operatorname{Soc}\left(Q_{1}\right)$ and $Q_{2} \neq \operatorname{Soc}\left(Q_{2}\right)$. Hence, $N / \operatorname{Soc}(N)$ has uniform dimension at least 2. Applying the same arguments to the module $Q_{2}$, and continuing the process in a similar manner, an obvious induction shows that $N / \operatorname{Soc}(N)$ has infinite uniform dimension, which is a contradiction to the fact that $N / \operatorname{Soc}(N)$ is Noetherian. This shows that $\operatorname{Soc}(N)$ is finitely generated, and therefore $N$ is Noetherian, completing our proof.

From Theorem 1 we obtain immediately the following consequence.

COROLlary 2 . Let $R$ be a ring such that every finitely generated right $R$-module is a direct sum of a projective module and a CS-module. Then $R$ is right Noetherian.

We now prove the following fact which will be crucial for the proof of our main result. Recall that a module $N$ in $\sigma[M]$ is called $\sum$-pure-injective in $\sigma[M]$ if every direct sum of copies of $M$ is pure-injective in $\sigma[M]$. A module $M$ is called pure semisimple if every module in the category $\sigma[M]$ is pure-injective. In this case, $\sigma[M]$ is called a pure semisimple category (see, for example, [13]).

Proposition 3. Let $M$ be a module and suppose that there is a cardinal number $\mathrm{c}$ such that every module in $\sigma[M]$ is a direct sum of c-generated modules. Then every module in $\sigma[M]$ is a direct sum of modules with local endomorphism rings.

Proof: It follows from Garcia and Martinez Hernandez [8] (see Garcia and Dung [7, Theorem 2.4]) that a pure-injective module $N$ in $\sigma[M]$ is $\Sigma$-pure-injective if and only if there is an infinite cardinal number $m$ such that the pure-injective envelope in $\sigma[M]$ of any direct sum of copies of $N$ is a direct sum of $\mathrm{m}$-generated modules. Hence, our hypothesis combined with this result implies that every pure-injective module in $\sigma[M]$ is $\Sigma$-pureinjective, hence is a direct sum of indecomposable modules with local endomorphism rings. This implies that $\sigma[M]$ is a pure semisimple category, so every module in $\sigma[M]$ is a direct sum of modules with local endomorphism rings (see, for example, [8]).

We are now in a position to prove the main result.

THEOREM 4 . Let $M$ be a right $R$-module satisfying (**). Then every module $N$ in $\sigma[M]$ has a decomposition $N=\bigoplus_{i \in I} N_{i}$, where for each $i \in I$, either $N_{i}$ is indecomposable projective or $N_{i}$ is uniform $\Sigma$-quasi-injective.

PROOF: First we show that there exists a cardinal number $\mathrm{c}$ such that each module $N \in \sigma[M]$ is a direct sum of c-generated modules. It follows from Theorem 1 that $M$ is a locally Noetherian module. Let $N$ be any module in $\sigma[M]$. By the condition $(* *)$, we have that $N=P \oplus K$, where $P$ is a projective module and $K$ is a CS-module. By Kaplansky's Theorem (see, for example, [1, Corollary 26.2]), $P$ is a direct sum of countably generated 
modules.

Note that $K$ is a locally Noetherian CS-module. Hence by [5, Corollary 8.3], $K$ has a decomposition $K=\bigoplus_{j \in J} K_{j}$, where each $K_{j}$ is an uniform module. For each $K_{j}$, we consider the $M$-injective envelope $E\left(K_{j}\right)$ of $K_{j}$ (that is, the injective envelope of $K_{j}$ in $\sigma[M]$ ). Since the category $\sigma[M]$ has a generating set consisting of finitely generated modules, clearly the collection of all isomorphism classes of uniform $M$-injective modules forms a set, implying that the collection of all isomorphism classes of uniform modules in $\sigma[M]$ is also a set. Hence there exists an infinite cardinal number $\mathrm{c}$ such that every uniform module in $\sigma[M]$ is c-generated. Therefore, the module $N$ in $\sigma[M]$ has a decomposition $N=\bigoplus_{i \in I} N_{i}$, where each $N_{i}$ is a c-generated module. By Proposition 3, we get that $\sigma[M]$ is a pure semisimple category, and so every module $N$ in $\sigma[M]$ is a direct sum of modules with local endomorphism rings.

Finally we show that every indecomposable direct summand of $N$ is projective or $\Sigma$-quasi-injective. Let $U$ be any indecomposable direct summand of $N$, and assume that $U$ is not projective. Consider the module $U^{(I)}$, where $I$ is any index set. By the condition (**), we know that $U^{(I)}=Q \oplus Y$ where $Q$ is projective and $Y$ is CS. If $Q \neq 0$, then by Azumaya's Theorem (see [1, Theorem 12.6]) $Q$ must contain a direct summand isomorphic to $U$. Hence $U$ is projective, which is a contradiction. This implies that $Q=0$, and so $U^{(I)}=Y$ is a CS-module. Hence, $U^{(I)}$ is a CS-module for each index set $I$, that is, $U$ is $\Sigma$-CS in the sense of [3] (see Clark and Wisbauer [2]). Now we shall use an argument in [4, Theorem 7, p.279] to show that $U$ is $\Sigma$-quasi-injective.

Let $V=\bigoplus_{i=1}^{\infty} U_{i}$, with $U_{i} \simeq U$ for all $i$. Because $V$ is a CS-module and $\operatorname{End}\left(U_{i}\right)$ is local for each $i$, the family $\left\{U_{i} \mid i \geqslant 1\right\}$ is locally semi-T-nilpotent (see [3, Theorem 2.4]). Let $\theta: U \rightarrow U$ be any monomorphism, and suppose that $\theta$ is not an isomorphism. By the locally semi-T-nilpotency of $\left\{U_{i} \mid i \geqslant 1\right\}$, it follows that, for any $x \in U$, there is a positive integer $n$ such that $\theta^{n}(x)=0$, which implies that $x=0$, a contradiction. Thus any monomorphism $\theta: U \rightarrow U$ is an isomorphism. Since $U \oplus U$ is CS, by [4, Lemma 3(b)], it follows that $U$ is $U$-injective, that is, $U$ is quasi-injective. It follows now from [5, Corollary 8.10] that $U$ is $\Sigma$-quasi-injective since $U$ is $\Sigma$-CS. This completes the proof.

The next result can also be derived from [10, Theorem 5] which was proved by different techniques.

PROPOSITION 5. If every right $R$-module is a direct sum of a projective module and a CS-module then $R$ is a right Artinian ring.

Proof: Under our hypothesis, it follows from the proof of Theorem 4 (for the case $M_{R}=R_{R}$ ) that there is a cardinal number $c$ such that every right $R$-module is a direct sum of c-generated modules. Hence, by [6, Theorem 20.23], $R$ is a right Artinian ring.

Finally, we consider the modules $M$ satisfying the property that every module $N \in$ $\sigma[M]$ is a direct sum of a projective module and a quasi-continuous module. 
THEOREM 6. The following conditions are equivalent for a right $R$-module $M$ :

(1) Every module $N \in \sigma[M]$ is a direct sum of a projective module and a quasi-continuous module;

(2) Every module $N \in \sigma[M]$ is a direct sum of a projective module and a quasi-injective module.

Proof: $(2) \Rightarrow(1)$ is clear.

(1) $\Rightarrow(2)$ Assume that (1) holds. Let $N$ be any module in $\sigma[M]$. Then $N=P \oplus K$, where $P$ is projective and $K$ is quasi-continuous. By Theorem 4, it follows that $K=$ $\bigoplus K_{i}$, where each $K_{i}$ is indecomposable, hence uniform. Without loss of generally we clearly may assume that each $K_{i}$ is non-projective. Thus, by Theorem 4 , each $K_{i}$ is quasi-injective. Because $K$ is quasi-continuous, it follows by [11, Theorem 2.13] that for each $j \in I,\left(\bigoplus_{i \neq j} K_{i}\right)$ is $K_{j}$-injective. Hence, by [11, Proposition 1.18], this implies that $K=\bigoplus_{i \in I} K_{i}$ is quasi-injective. Therefore, $N$ is a direct sum of a projective module and a quasi-injective module.

We conclude the paper with some remarks.

REMARKS.

(a) The results in this paper remain true (with similar arguments) if the conditions $(*)$ and $(* *)$ are replaced by the weaker ones that every (finitely generated) module in $\sigma[M]$ is a direct sum of a module which is projective in $\sigma[M]$ and a CS-module.

(b) Rings satisfying the property that every right $R$-module is a direct sum of a projective module and a quasi-injective module have recently been studied by Huynh and Rizvi [10]. We refer to this work for several characterisations and ideal-theoretic descriptions of these rings.

\section{REFERENCES}

[1] F.W. Anderson and K.R. Fuller, Rings and categories of modules (Springer-Verlag, Berlin, Heidelberg, New York, 1974).

[2] J. Clark and R. Wisbauer, ' $\sum$-extending modules', J. Pure Appl. Algebra 104 (1995), 19-32.

[3] N.V. Dung, 'On indecomposable decompositions of CS-modules II', J. Pure Appl. Algebra 119 (1997), 139-153.

[4] N.V. Dung and P.F. Smith, 'Rings for which certain modules are CS', J. Pure Appl. Algebra 102 (1995), 273-287.

[5] N.V. Dung, D.V. Huynh, P.F. Smith and R. Wisbauer, Extending modules, Pitman Research Notes in Mathematics Series 313 (Longman, Harlow, 1994).

[6] C. Faith, Algebra II: Ring theory (Springer-Verlag, Berlin, Heidelberg, New York, 1976).

[7] J.L. Garcia and N.V. Dung, 'Some decomposition properties of injective and pure-injective modules', Osaka J. Math. 31 (1994), 95-108. 
[8] J.L. Garcia and J. Martinez Hernandez, 'Purity through Gabriel's functor rings', Bull. Soc. Math. Belgique 31 (1994), 95-108.

[9] D.V. Huynh, S.T. Rizvi and M.F. Yousif, 'Rings whose finitely generated modules are extending', J. Pure Appl. Algebra 111 (1996), 325-328.

[10] D.V. Huynh and S.T. Rizvi, 'On some classes of artinian rings', J. Algebra (to appear).

[11] S.H. Mohamed and B.J. Müller, Continuous and discrete modules, London Math. Soc. Lecture Notes 147 (Cambridge Univ. Press., Cambridge, 1990).

[12] N. Vanaja, 'All finitely generated $M$-subgenerated modules are extending', Comm. $A l$ gebra 24 (1996), 543-572.

[13] R. Wisbauer, Foundations of module and ring theory (Gordon and Breach, Philadelphia, P.A., 1991).

Department of Mathematics

Naresuan Unviersity

Phitsanulok 65000

Thailand

e-mail: somyotp@nu.ac.th 